\title{
On Borsuk's Conjecture for Two-Distance Sets
}

\author{
Andriy Bondarenko
}

Received: 27 September 2013 / Revised: 16 December 2013 / Accepted: 17 February 2014 /

Published online: 5 March 2014

(C) Springer Science+Business Media New York 2014

\begin{abstract}
In this paper, we answer Larman's question on Borsuk's conjecture for two-distance sets. We find a two-distance set consisting of 416 points on the unit sphere $S^{64} \subset \mathbb{R}^{65}$ which cannot be partitioned into 83 parts of smaller diameter. This also reduces the smallest dimension in which Borsuk's conjecture is known to be false. Other examples of two-distance sets with large Borsuk numbers are given.
\end{abstract}

Keywords Borsuk's conjecture · Two-distance sets · Strongly regular graphs

\section{Introduction}

For each $n \in \mathbb{N}$ the Borsuk number $b(n)$ is the minimal number such that any bounded set in $\mathbb{R}^{n}$ consisting of at least 2 points can be partitioned into $b(n)$ parts of smaller diameter. In 1933, Karol Borsuk [4] conjectured that $b(n)=n+1$. The conjecture was disproved by Kahn and Kalai [13] who showed that, in fact, $b(n)>1.2^{\sqrt{n}}$ for large $n$. In particular, their construction implies that $b(n)>n+1$ for $n=1325$ and for all $n>2014$. This result attracted a substantial amount of attention from many mathematicians; see, for example, [1, 2, 5, 21]. Improvements on the smallest dimension $n$ such that $b(n)>n+1$ were obtained by Nilli [17] $(n=946)$, Raigorodskii [20]

This work was carried out during the tenure of an ERCIM "Alain Bensoussan" Fellowship Programme. The research leading to these results has received funding from the European Union Seventh Framework Programme (FP7/2007-2013) under grant agreement $n^{\circ} 246016$.

A. Bondarenko

Department of Mathematical Sciences, Norwegian University of Science and Technology,

7491 Trondheim, Norway

e-mail: andriybond@gmail.com

A. Bondarenko

Department of Mathematical Analysis, Taras Shevchenko National University of Kyiv, str. Volodymyrska, 64, Kyiv, 01033, Ukraine 
$(n=561)$, Weißbach [22] $(n=560)$, Hinrichs [10] $(n=323)$, and Pikhurko [19] $(n=321)$. Until recently the best known result was that Borsuk's conjecture is false for $n \geq 298$; see [11]. On the other hand, many related problems are still unsolved. Borsuk's conjecture can be wrong even in dimension 4 . Only the estimate $b(4) \leq 9$ is known; see [15].

In the 1970s, Larman asked if Borsuk's conjecture is true for two-distance sets; see also [14] and [21]. Denote by $b_{2}(n)$ the Borsuk number for two-distance sets in dimension $n$, that is, the minimal number such that any two-distance set in $\mathbb{R}^{n}$ can be partitioned into $b_{2}(n)$ parts of smaller diameter. The aim of this paper is to construct two-distance sets with large Borsuk numbers. Two basic constructions follow from Euclidean representations of certain strongly regular graphs. First we prove

Theorem 1 There is a two-distance subset $\left\{x_{1}, \ldots, x_{416}\right\}$ of the unit sphere $S^{64} \subset \mathbb{R}^{65}$ such that $\left\langle x_{i}, x_{j}\right\rangle=1 / 5$ or $-1 / 15$ for $i \neq j$ which cannot be partitioned into 83 parts of smaller diameter.

Hence $b(65) \geq b_{2}(65) \geq 84$. We also prove the following

Theorem 2 There is a two-distance subset $\left\{x_{1}, \ldots, x_{31671}\right\}$ of the unit sphere $S^{781}$ such that $\left\langle x_{i}, x_{j}\right\rangle=1 / 10$ or $-1 / 80$ for $i \neq j$ which cannot be partitioned into 1376 parts of smaller diameter.

Then, using the configurations from Theorem 1 and Theorem 2, we prove

Corollary 1 For integers $n \geq 1$ and $k \geq 0$, we have

$$
b_{2}(66 n+k) \geq 84 n+k+1,
$$

and

$$
b_{2}(783 n+k) \geq 1377 n+k+1 .
$$

Finally, using again the configuration from Theorem 2, we prove slightly better estimates for $b_{2}(781), b_{2}(780)$, and $b_{2}(779)$ than what can be obtained from (1).

Corollary 2 The following inequalities hold:

$$
b_{2}(781) \geq 1225, \quad b_{2}(780) \geq 1102, \quad \text { and } \quad b_{2}(779) \geq 1002 .
$$

The paper is organized as follows. First, in Sect. 2, we describe Euclidean representations of strongly regular graphs by two-distance sets and then, in Sect. 3, we prove our main results.

\section{Euclidean Representations of Strongly Regular Graphs}

A strongly regular graph $\Gamma$ with parameters $(v, k, \lambda, \mu)$ is an undirected regular graph on $v$ vertices of valency $k$ such that each pair of adjacent vertices has $\lambda$ common 
neighbors, and each pair of nonadjacent vertices has $\mu$ common neighbors. The adjacency matrix $A$ of $\Gamma$ has the following properties:

$$
A J=k J
$$

and

$$
A^{2}+(\mu-\lambda) A+(\mu-k) I=\mu J
$$

where $I$ is the identity matrix and $J$ is the matrix with all entries equal to 1 of appropriate sizes. These conditions imply that

$$
(v-k-1) \mu=k(k-\lambda-1) .
$$

Moreover, the matrix $A$ has only 3 eigenvalues: $k$ of multiplicity 1 , one positive eigenvalue

$$
r=\frac{1}{2}\left(\lambda-\mu+\sqrt{(\lambda-\mu)^{2}+4(k-\mu)}\right)
$$

of multiplicity

$$
f=\frac{1}{2}\left(v-1-\frac{2 k+(v-1)(\lambda-\mu)}{\sqrt{(\lambda-\mu)^{2}+4(k-\mu)}}\right),
$$

and one negative eigenvalue

$$
s=\frac{1}{2}\left(\lambda-\mu-\sqrt{(\lambda-\mu)^{2}+4(k-\mu)}\right)
$$

of multiplicity

$$
g=\frac{1}{2}\left(v-1+\frac{2 k+(v-1)(\lambda-\mu)}{\sqrt{(\lambda-\mu)^{2}+4(k-\mu)}}\right) .
$$

Clearly, both $f$ and $g$ must be integers. This, together with (3), gives a collection of feasible parameters $(v, k, \lambda, \mu)$ for strongly regular graphs.

Let $V$ be the set of vertices $\Gamma$. Consider the columns $\left\{y_{i}: i \in V\right\}$ of the matrix $A-s I$ and put $x_{i}:=z_{i} /\left\|z_{i}\right\|$, where

$$
z_{i}=y_{i}-\frac{1}{v} \sum_{j \in V} y_{j}, \quad i \in V .
$$

Note that while the vectors $x_{i}$ lie in $\mathbb{R}^{v}$, they span an at most $f$-dimensional vector space. Thus for convenience we consider them to lie in $\mathbb{R}^{f}$. By easy calculations

$$
\left\langle x_{i}, x_{j}\right\rangle= \begin{cases}1 & \text { if } i=j \\ p & \text { if } i \text { and } j \text { are adjacent } \\ q & \text { otherwise }\end{cases}
$$


where

$$
\begin{aligned}
& p=\frac{\lambda-2 s-\beta}{s^{2}+k-\beta}, \quad q=\frac{\mu-\beta}{s^{2}+k-\beta}, \\
& \beta=\frac{1}{v}\left(s^{2}+k+k(\lambda-2 s)+(v-k-1) \mu\right) .
\end{aligned}
$$

Denote by $\Gamma_{f}$ the configuration $x_{i}, i \in V$. Similarly, we can define the configuration $\Gamma_{g}$ in $\mathbb{R}^{g}$. The configurations $\Gamma_{f}$ and $\Gamma_{g}$ were also considered in [8] and have many fascinating properties. For example, they are spherical 2-designs.

\section{Proof of Main Results}

For a graph $G$, denote by the clique covering number $c c(G)$ the smallest number of cliques needed to cover the vertex set of $G$. Trivially, $c c(G) \geq v / m$, where $v$ is the number of vertices and $m$ is the maximal size of a clique in $G$. First, we prove the following simple statement.

Proposition 1 For any strongly regular graph $\Gamma$, the configuration $\Gamma_{f}$ in $\mathbb{R}^{f}$ cannot be partitioned into fewer parts of smaller diameter than $\operatorname{cc}(G)$.

Proof We have by (5) that $\lambda-\mu-2 s>0$. Therefore, by (6), $p-q>0$, and hence the diameter of $\Gamma_{f}$ corresponds to the distance between non-edges.

Remark 1 We did an extensive search of counterexamples to Borsuk's conjecture applying Proposition 1 for various known strongly regular graphs. We were able to find two very nice strongly regular graphs that give counterexamples in dimensions 65 and 782, respectively. Probably the first example has the smallest possible dimension for this approach. However, we cannot prove this because the complete description of all strongly regular graphs is known only for very few parameter sets.

For any vertex $v \in V$ of a strongly regular graph $\Gamma$, let $N(v)$ be the set of all neighbors of $v$ and let $N^{\prime}(v)$ be the set of non-neighbors of $v$, i.e., $N^{\prime}(v)=V \backslash(\{v\} \cup$ $N(v))$.

Proof of Theorem 1 We consider the configuration $\Gamma_{f}$ of the well-known strongly regular graph $\Gamma=G_{2}(4)$ with parameters $(416,100,36,20)$. By (4), we have that $f=65$. Moreover, by (6), $p=1 / 5$ and $q=-1 / 15$. Therefore, by Proposition 1 , it is enough to show that $\Gamma$ has no 6-clique. We will use the following theorem consisting of four independent results that can be found in [6].

\section{Theorem A}

(i) For each $u \in V$, the subgraph of $\Gamma$ induced on $N(u)$ is a strongly regular graph with parameters $(100,36,14,12)$ (the Hall-Janko graph). In other words, the Hall-Janko graph is the first subconstituent of $\Gamma$. 
(ii) The first subconstituent of the Hall-Janko graph is the $U_{3}(3)$ strongly regular graph with parameters $(36,14,4,6)$.

(iii) The first subconstituent of $U_{3}(3)$ is a graph on 14 vertices of regularity 4 (the co-Heawood graph).

(iv) The co-Heawood graph has no triangles.

Parts (i)-(iii) are folklore. They follow from D.G. Higman's theory of rank 3 permutation groups (see also [9] and [16]). Part (iv) follows from the fact that the coHeawood graph is a subgraph of the Gewirtz graph with parameters $(56,10,0,2)$; see also [3].

Now, for vertices $u, v, w \in V$ forming a triangle, (i)-(iii) imply that

$$
|N(u) \cap N(v) \cap N(w)|=14 .
$$

Moreover, the subgraph induced on $N(u) \cap N(v) \cap N(w)$ is the co-Heawood graph. Therefore, by (iv), the maximal cliques in $\Gamma$ are of size 5 .

Remark 2 Very recently Jenrich [12] found a subconfiguration of $\Gamma_{f}$ consisting of 352 points that spans only a 64-dimensional subspace. This immediately implies that $b_{2}(64) \geq 71$. Then the author used a computer search to prove that actually this configuration gives $b_{2}(64) \geq 72$.

Proof of Theorem 2 Consider the configuration $\Gamma_{f}$ of the $F i_{23}$ graph with parameters $(31671,3510,693,351)$. We have $f=782, p=1 / 10$, and $q=-1 / 80$. Hence, by Proposition 1, it is enough to prove that the size of a clique in $\Gamma$ is at most 23 (we need at least $31671 / 23=1377$ parts). We will use the well-known fact (see [18]) that the first subconstituent of $\Gamma$ is the strongly regular graph with parameters $(3510,693,180,126)$ and the second subconstituent of $\Gamma$ is the strongly regular graph $G$ with parameters $(693,180,51,45)$. Note that the Hoffman bound [7] implies that the size of a clique in $G$ is at most 21 . We will also give a very short selfcontained proof of this estimate. Indeed, consider the complement graph $\bar{G}$ having parameters $(693,512,376,384)$. For the configuration $\bar{G}_{f}$, we have that $f=440$, $p=1 / 64$, and $q=-1 / 20$. Now the required estimate follows from the fact that for each clique $K$ in $G$ and vectors $x_{i} \in \bar{G}_{f}$

$$
\left\langle\sum_{i \in K} x_{i}, \sum_{i \in K} x_{i}\right\rangle=|K|+|K|(|K|-1) q \geq 0 .
$$

Therefore, the size of a clique in $\Gamma$ is at most 23 .

Proof of Corollary 1 Let us first prove (1) for $k=0$. Fix $n \in \mathbb{N}$ and put $m=66 n$. Consider the following coordinate representation of a vector $y \in \mathbb{R}^{m}$ :

$$
y=\left(y_{1}, \ldots, y_{n} \mid a_{1}, \ldots, a_{n}\right),
$$

where $y_{k} \in \mathbb{R}^{65}$ and $a_{k} \in \mathbb{R}, k=1, \ldots, n$. Now we take the following set of unit vectors in $\mathbb{R}^{m}: Y=\left\{v_{i k}, i=1, \ldots, 416, k=1, \ldots, n\right\}$, where 


$$
\begin{aligned}
v_{i k} & =\left(0, \ldots, 0, \sqrt{15} / 4 x_{i}, 0, \ldots, 0 \mid 0, \ldots, 0,1 / 4,0, \ldots, 0\right), \\
i & =1, \ldots, 416, k=1, \ldots, n .
\end{aligned}
$$

Here each $v_{i k}$ has only two nonzero coordinates $y_{k}$ and $a_{k}$, and vectors $x_{i}$ are such as in Theorem 1. Clearly, $\left\langle v_{i k}, v_{j l}\right\rangle=0$ if $k \neq l$. Moreover,

$$
\left\langle v_{i k}, v_{j k}\right\rangle= \begin{cases}1 & \text { if } i=j \\ 1 / 4 & \text { if } i \text { and } j \text { are adjacent } \\ 0 & \text { otherwise. }\end{cases}
$$

Therefore, $Y$ is a two-distance set consisting of $416 n$ vectors. Now, by Theorem 1 , this set cannot be partitioned into fewer than $84 n$ parts of smaller diameter. Now we can add to the set the vector $v$ which is at distance $\sqrt{2}$ to each vector of $Y$, where

$$
v=(0, \ldots, 0 \mid \alpha, \ldots, \alpha), \quad \text { and } \quad \alpha=\frac{1+\sqrt{1+16 n}}{4 n}
$$

is a solution of the equation $(\alpha-1 / 4)^{2}+(n-1) \alpha^{2}=17 / 16$. This implies that $b_{2}(m) \geq 84 n+1$. Finally, we note that all these $416 n+1$ vectors are at the same distance $R$ to the vector $(0, \ldots, 0 \mid \gamma, \ldots, \gamma)$, where

$$
R=\frac{4 \sqrt{n}}{\sqrt{16 n+1}}<1 \quad \text { and } \quad \gamma=\frac{\alpha}{4 n \alpha-1}
$$

is a solution of the equation $(\gamma-1 / 4)^{2}+(n-1) \gamma^{2}+15 / 16=n(\alpha-\gamma)^{2}$. Hence we can add a new vector at the diameter distance $\sqrt{2}$ to each of these $416 n+1$ vectors to get a new set of $416 n+2$ vectors in $\mathbb{R}^{m+1}$ provided that $b_{2}(m+1) \geq 84 n+2$. We can also rescale this new set to be on the sphere $S^{m}$. Now inductive application of this procedure immediately gives us (1). This procedure was also described in [11, Lemma 9]. Similarly, Theorem 2 implies (2).

Proof of Corollary 2 Let $\Gamma$ be the $F i_{23}$ graph. For a vertex $u \in V$, consider the subset $\left\{x_{i}: i \in N^{\prime}(u)\right\}$ of the configuration $\Gamma_{f}$. This subset lies in the hyperplane $\left\langle x_{u}, x\right\rangle=-1 / 80$ and consists of $31671-3510-1=28160$ vectors. Hence, $b_{2}(781)>[28160 / 23]=1224$.

Similarly, for adjacent vertices $u$ and $v$, the subset $\left\{x_{i}: i \in N^{\prime}(u) \cap N^{\prime}(v)\right\}$ consists of $31671-2 \times 3510+693=25344$ vectors. This subset lies in the hyperplane $\{x \in$ $\mathbb{R}^{782}:\left\langle x_{u}, x\right\rangle=-1 / 80$ and $\left.\left\langle x_{v}, x\right\rangle=-1 / 80\right\}$, and hence $b_{2}(780)>[25344 / 23]=$ 1101 .

Finally, consider a subset $\left\{x_{i}: i \in N^{\prime}(u) \cap N^{\prime}(v) \cap N^{\prime}(w)\right\}$ such that the vertices $u, v, w$ form a triangle. This subset consists of $31671-3 \times 3510+3 \times 693-180=$ 23040 vectors, and hence $b_{2}(779)>[23040 / 23]=1001$.

Acknowledgements The author thanks Noga Alon, Danylo Radchenko, and Kristian Seip for fruitful discussions. The author also wishes to thank the Centre for Advanced Study at the Norwegian Academy of Science and Letters in Oslo and Mathematisches Forschungsinstitut Oberwolfach for their hospitality during the preparation of this manuscript and for providing a stimulating atmosphere for research. 


\section{References}

1. Aigner, M., Ziegler, G.M.: Proofs from THE BOOK, 4th edn. Springer, Berlin, New York (2009)

2. Alon, N.: Discrete mathematics: methods and challenges. In: Proceedings of the International Congress of Mathematicians, Beijing, 2002, vol. 1, pp. 119-135. Higher Ed. Press, Beijing (2002)

3. Bamberg, J., de Clerck, F., Durante, N.: Intriguing sets in partial quadrangles. J. Comb. Des. 19, 217-245 (2011)

4. Borsuk, K.: Three theorems on the $n$-dimensional sphere. Fundam. Math. 20, 177-190 (1933) (in German)

5. Brass, P., Moser, W., Pach, J.: Research Problems in Discrete Geometry. Springer, Berlin (2005)

6. Brouwer, A.: A slowly growing collection of graph descriptions. published electronically. http://www. win.tue.nl/ aeb/graphs/index.html

7. Brouwer, A.E., Cohen, A.M., Neumaier, A.: Distance-Regular Graphs. Springer, Berlin (1989)

8. Cameron, P.: Strongly regular graphs. In: Topics in Algebraic Graph Theory, pp. 203-221. Cambridge Univ. Press, Cambridge (2004)

9. Griess, R.: Twelve Sporadic Groups. Springer Monographs in Mathematics. Springer, Berlin (1998)

10. Hinrichs, A.: Spherical codes and Borsuk's conjecture. Discrete Math. 243, 253-256 (2002)

11. Hinrichs, A., Richter, C.: New sets with large Borsuk numbers. Discrete Math. 270, 137-147 (2003)

12. Jenrich, T.: A 64-dimensional two-distance counterexample to Borsuk's conjecture. arXiv:1308.0206

13. Kahn, J., Kalai, G.: A counterexample to Borsuk's conjecture. Bull. Am. Math. Soc. 29, 60-62 (1993)

14. Kalai, G.: Around Borsuk's conjecture. published electronically at http://gilkalai.wordpress.com

15. Lassak, M.: An estimate concerning Borsuk's partition problem. Bull. Acad. Pol. Sci., Ser. Math. 30, 449-451 (1982)

16. Leemans, D.: A family of geometries related to the Suzuki tower. Commun. Algebra 33, 2201-2217 (2005)

17. Nilli, A.: On Borsuk's problem. In: Jerusalem Combinatorics, 1993, pp. 209-210. Amer. Math. Soc., Providence (1994)

18. Pasechnik, D.: Geometric characterization of the sporadic groups $F i_{22}, F i_{23}$, and $F i_{24}$. J. Comb. Theory, Ser. A 68, 100-114 (1994)

19. Pikhurko, O.: Borsuk's conjecture fails in dimensions 321 and 322 (2002). arXiv:math.CO/0202112

20. Raigorodskii, A.M.: On dimensionality in the Borsuk problem. Usp. Mat. Nauk 52, 181-182 (1997) (in Russian). English translation in Russian Math. Surveys 52, 1324-1325 (1997)

21. Raigorodskii, A.M.: Around Borsuk's hypothesis. J. Math. Sci. 154, 604-623 (2008)

22. Weißbach, B.: Sets with large Borsuk number. Beiträge Algebra Geom. 41, 417-423 (2000) 